\title{
ON THE UNION OF SETS OF SYNTHESIS AND DITKIN'S CONDITION IN REGULAR BANACH ALGEBRAS
}

\author{
BY AHARON ATZMON
}

The questions have been raised, whether for every commutative semisimple regular Banach algebra with unit, the union of two sets of synthesis is again a set of synthesis, whether every set of synthesis is a Ditkin set, and whether a singleton which is of synthesis is a Ditkin set (cf. [4, pp. 31, 34, 41] and [2, p. 225]). The purpose of this note is to show that for the Banach algebra obtained by adjoining a unit to the Banach algebra introduced by Mirkil in [3], the answer to all three questions is in the negative. In fact Mirkil's proof of his main result $[3, \S 5]$ contains implicitly a negative answer to the third (and therefore also to the second) question. Mirkils algebra $\mathbf{M}$ is defined as follows: Let $\mathbf{T}$ denote the circle group identified with the interval $[-\pi, \pi)$, the group operation being addition modulo $2 \pi$. $\mathbf{M}$ is the convolution algebra of all functions in $L^{2}(\mathrm{~T})$ which are continuous on the interval $[-\pi / 2, \pi / 2]$ with norm defined by

$$
\|f\|=\|f\|_{2}+\sup \{|f(t)|:|t| \leqslant \pi / 2\} \text {. }
$$

(We recall that the convolution of two functions, $f, g$ in $L^{2}(\mathrm{~T})$ is defined by

$$
\left.f * g(t)=\frac{1}{2 \pi} \int_{\mathbf{T}} f(t-x) g(x) d x, t \in T .\right)
$$

As shown in [3], $\mathbf{M}$ is a Banach algebra in which the trigonometric polynomials are dense, whose regular maximal ideal space can be identified with the set of integers $\mathbf{Z}$, and its Gelfand representation is given by the Fourier transform $f \rightarrow \hat{f}$ where $\hat{f}(n)=(1 / 2 \pi) \int_{\mathbf{T}} f(x) e^{-i n x} d x, n \in \mathbf{Z}$.

We shall denote by $\mathbf{M}_{1}$ the Banach algebra obtained by the standard adjunction of unit to $\mathbf{M}\left(\mathbf{M}_{1}\right.$ can also be regarded as the convolution algebra $M \oplus \mathbf{D}$ where $\mathbf{D}$ is the one dimensional vector space spanned by the unit point measure concentrated at $\tau$ ). The maximal ideal space of $\mathbf{M}_{1}$ can be identified with $\mathbf{Z} \cup$ $\{\infty\}$, the one point compactification of $\mathbf{Z}$.

We refer to $[2$, p. 230] for the definitions of sets of synthesis for regular Banach algebras (see also $[4$, p. 28] where these sets are called Wiener sets). We shall give here the definition directly for the algebras $\mathbf{M}$ and $\mathbf{M}_{\mathbf{1}}$.

Let $\mathbf{M}^{*}$ denote the dual space of $\mathbf{M}$. For every $\nu$ in $M^{*}$ we set $\hat{\nu}(n)=$

Received by the editors June $26,1979$.

AMS (MOS) subject classifications (1970). Primary 43A45; Secondary 46J20. 
$\left\langle e^{i n x}, v\right\rangle, n \in \mathbf{Z}$, and define the spectrum of $\nu$ to be the set

$$
\sigma(\nu)=\{n \in \mathbf{Z}: \hat{v}(n) \neq 0\} .
$$

Every function $g$ in $L^{2}(\mathbf{T})$ defines an element of $\mathbf{M}^{*}$ by the mapping

$$
f \rightarrow\langle f, g\rangle=\frac{1}{2 \pi} \int_{\mathrm{T}} f(x) \overline{g(x)} d x, \quad f \in \mathbf{M} .
$$

We shall denote this element also by $g$.

Definition 1. A set $E \subset \mathbf{Z}$ is called a set of synthesis for $\mathbf{M}$ if every element in $\mathbf{M}^{*}$ with spectrum contained in $E$, is in the $w^{*}$ closure of the vector space spanned in $\mathbf{M}^{*}$ by the set $\left\{e^{i n x}: n \in E\right\}$. By the Hahn-Banach theorem, this is equivalent to the requirement that $\langle f, \nu\rangle=0$ for every pair $\nu \in \mathbf{M}^{*}$ and $f \in \mathbf{M}$ such $\sigma(\nu) \subset E$ and $\hat{f}=0$ on $E$.

DEFINITION 2. A closed set $K \subset \mathbf{Z} \cup\{\infty\}$ is called a set of synthesis for $\mathbf{M}_{\mathbf{1}}$ if the set $K \cap \mathbf{Z}$ is of synthesis for $\mathbf{M}$.

Since the trigonometric polynomials are dense in $\mathbf{M}$, the empty set is of synthesis for $\mathbf{M}$, and therefore by Definition 2, the singleton $\{\infty\}$ is of synthesis for $\mathbf{M}_{1}$. (It is easy to see that the usual definition of sets of synthesis for regular Banach algebras given in $\left[2\right.$, p. 230] coincides, for the algebra $\mathbf{M}_{1}$, with Definition 2.)

It is clear that the algebra $\mathbf{M}$ is semisimple and regular and therefore the same is true for the algebra $\mathbf{M}_{\mathbf{1}}$.

We refer to $[4$, p. 30] for the definition of Ditkin sets (called there WienerDitkin sets) for regular Banach algebras. We only note here that the condition for $\{\infty\}$ to be a Ditkin set for $\mathbf{M}_{1}$, is equivalent to the requirement that for every function $f$ in $\mathbf{M}$ there exists a sequence of trigonometric polynomials $\left(p_{n}\right)_{n=1}^{\infty}$ such that $p_{n} * f \rightarrow f$ in the norm of $\mathbf{M}$.

In what follows, we denote for every pair of integers $r$ and $s$ by $r Z+s$ the set of all integers of the form $r n+s, n \in \mathbf{Z}$. The negative answers to the questions mentioned, is contained in the following.

Theorem. (a) The sets $K_{1}=4 \mathrm{Z} \cup\{\infty\}$ and $K_{2}=4 \mathrm{Z}+2 \cup\{\infty\}$ are of synthesis for $\mathbf{M}_{1}$ but the set $K_{1} \cup K_{2}$ is not of synthesis for $\mathbf{M}_{1}$.

(b) The singleton $\{\infty\}$ is not a Ditkin set for $\mathbf{M}_{1}$.

Proof of (a). We have to show that the sets $E_{1}=4 \mathrm{Z}$ and $E_{2}=4 \mathrm{Z}+2$ are of synthesis for $\mathbf{M}$ but the set $2 \mathbf{Z}=E_{1} \cup E_{2}$ is not of synthesis for $\mathbf{M}$. For this we need first to identify the dual space $M^{*}$. Let $C[-\pi / 2, \pi / 2]$ denote as usual the Banach space of complex continuous functions on $[-\pi, \pi / 2]$ with the sup norm, and consider the Banach space $\mathrm{B}=L^{2}(\mathrm{~T}) \times C[-\pi / 2, \pi / 2]$ with norm $\|(f, h)\|=\|f\|_{2}+\|h\|_{C[-\pi / 2, \pi / 2]}$. Noticing that the mapping

$$
f \rightarrow\left(f, f_{\mid[-\pi / 2, \pi / 2]}\right), \quad f \in \mathbf{M}
$$


(where $f_{\mid[-\pi / 2, \pi / 2]}$ denotes the restriction of $f$ to $[-\pi / 2, \pi / 2]$ ) is an isometric isomorphism of $\mathbf{M}$ onto a closed subspace of $\mathbf{B}$, using the Hahn Banach theorem, the fact that $\mathbf{B}^{*}=\left(L^{2}(\mathrm{~T})\right)^{*} \times C^{*}[-\pi / 2, \pi / 2]$, and the known representations of the dual spaces of $L^{2}(\mathrm{~T})$ and $C[-\pi / 2, \pi / 2]$, we see that every element $\nu$ in $\mathbf{M}^{*}$ can be represented by a Borel measure on $\mathbf{T}$ (which we also denote by $\nu$ ) which admits a decomposition of the form $\nu=g d x+\mu$ where $g \in L^{2}(\mathrm{~T})$ and $\mu$ is a Borel measure supported on $[-\pi / 2, \pi / 2]$, and the action of $\nu$ on $\mathbf{M}$ is given by

$$
\langle f, \nu\rangle=\frac{1}{2 \pi} \int_{\mathbf{T}} f(x) \overline{g(x)} d x+\int_{\mathbf{T}} f(x) d \bar{\mu}(x), \quad f \in \mathbf{M} .
$$

Clearly $\|\nu\|_{\mathbf{M}^{*}} \leqslant \max \left\{\|g\|_{2},|\mu|(\mathbf{T})\right\}$ where $|\mu|(\mathbf{T})$ is the total variation of $\mu$. Suppose now that $\nu=g d x+\mu \in \mathbf{M}^{*}$ with $g$ and $\mu$ as described above, and assume that $\sigma(\nu) \subset E_{1}=4 \mathrm{Z}$. Then $\hat{\nu}(n)=\int_{\mathrm{T}} e^{-i n t} d \nu(t)=0$ for $n \notin 4 \mathrm{Z}$ and therefore for every trigonometric polynomial $p$

$$
\int_{\mathbf{T}} p\left(t+\frac{\pi}{2}\right) d \nu(t)=\int_{\mathbf{T}} p(t) d \nu(t)
$$

and since a continuous function on $\mathbf{T}$ is the uniform limit of trigonometric polynomials, the equality remains true if $p$ is replaced by any such function. This shows that $\nu$ is a measure of period $\pi / 2$, that is, $\nu(S+\pi / 2)=\nu(S)$ for every Borel set $S \subset \mathrm{T}$. On the other hand, since $\mu$ is supported on $[-\pi / 2, \pi / 2], \nu(S)=$ $\int_{S} \overline{g(x)} d x$ for every Borel set $S \subset[0, \pi / 2)$. Combining these facts we see that $\nu$ is in $L^{2}(\mathrm{~T})$, and therefore setting $S_{N}(\nu)=\Sigma_{n=-N}^{N} \hat{\nu}(n) e^{i n x}, N=0,1, \ldots$, we deduce that

$$
\left\|\nu-S_{N}(\nu)\right\|_{\mathrm{M}^{*}} \leqslant\left\|\nu-S_{N}(\nu)\right\|_{2} \rightarrow 0 \quad \text { as } N \rightarrow \infty .
$$

This shows that every element in $\mathbf{M}^{*}$ with spectrum contained in $E_{1}$, is even in the norm closure in $\mathbf{M}^{*}$, of the vector space spanned in $\mathbf{M}^{*}$ by the $\operatorname{set}\left\{e^{i n x}\right.$ : $\left.n \in E_{1}\right\}$, and therefore $E_{1}$ is of synthesis for M. To show that $E_{2}=4 \mathrm{Z}+2$ is also a set of synthesis for $\mathbf{M}$, consider a measure $\rho$ in $\mathbf{M}^{*}$ with spectrum in $E_{2}$; then the spectrum of the measure $e^{2 i x} \rho$ is in $E_{1}$, hence by the previous part of the proof this measure is in $L^{2}(\mathrm{~T})$, and therefore $\rho$ is also in $L^{2}(\mathrm{~T})$. This implies as before, that $E_{2}$ is of synthesis for $\mathbf{M}$ (even in the norm topology of $\mathbf{M}^{*}$ ). The remaining assertions of the theorem follow from the main result in [3]. For the sake of completeness we include here a different proof which is more in line with the approach of this paper. To show that the set $E=E_{1} \cup E_{2}$ is not of synthesis for $\mathbf{M}$, consider the element of $\mathbf{M}^{*}$ defined by the measure $\mu=\delta_{\pi / 2}+\delta_{-\pi / 2}$ (for every $\tau \in \mathrm{T}, \delta_{\tau}$ denotes the unit point measure concentrated at $\tau$ ) and the function $f$ in $M$ defined by: $f(x)=1$ for $|x| \leqslant \pi / 2$ and $f(x)=-1$ for $\pi / 2<$ $|x| \leqslant \pi$. Then $\hat{\mu}(n)=2 \cos (n \pi / 2), \forall n \in \mathbf{Z}, \hat{f}(n)=2 \sin (n \pi / 2) / n \pi$, for $n \in \mathbf{Z} \backslash\{0\}$, and $\hat{f}(0)=0$. Thus $\sigma(\mu) \subset E$ and $\hat{f}=0$ on $E$, but $\langle f, \mu\rangle=2$, and therefore $E$ is not a set of synthesis for $M$. 
Proof of (b). To show that $\{\infty\}$ is not a Ditkin set for $\mathbf{M}_{1}$, consider again the measure $\mu$ and the function $f$ defined above. Noticing that $\langle q, \mu\rangle=0$ for every trigonometric polynomial $q$ such that $\hat{q}=0$ on $2 Z$, we obtain for every trigonometric polynomial $p$ (by using the identity $p * f=\Sigma_{n=-N}^{N} \hat{p}(n) \hat{f}(n) e^{i n x}$, where $N$ is the degree of $p$ ) that

$$
\|f-p * f\|_{\mathrm{M}} \geqslant\|\mu\|_{\mathrm{M}}^{-1}\langle f-p * f, \mu\rangle=1
$$

and consequently $\{\infty\}$ is not a Ditkin set for $\mathbf{M}_{1}$.

REMARK. The proof of the theorem shows that the sets $K_{1}$ and $K_{2}$ are of synthesis for $\mathbf{M}_{1}$ even in the norm topology of $\mathbf{M}_{1}^{*}$ but $K_{1} \cup K_{2}$ is not of synthesis for $\mathbf{M}_{1}$ even in the $w^{*}$ topology of $M_{1}$.

The answers to the first and second questions mentioned at the beginning are not known for group algebras of locally compact noncompact abelian groups; in particular they are not known for the group algebra of $\mathbf{Z}$. A discussion of these problems and partial results can be found in [1, Chapter 1 and 2], [4, Chapters 2 and 6] and [5, Chapter 7].

\section{REFERENCES}

1. J. Benedetto, Spectral synthesis, B. G. Teubner, Stuttgart, 1975.

2. Y. Katznelson, An introduction to harmonic analysis, Wiley, New York, 1968.

3. H. Mirkil, A counterexample to discrete spectral synthesis, Composito Math. 14 (1960), 269-273.

4. H. Reiter, Classical harmonic analysis and locally compact groups, Calderon Press, Oxford, 1968.

5. W. Rudin, Fourier analysis on groups, Interscience, New York, 1962.

DEPARTMENT OF MATHEMATICS, TECHNION-ISRAEL INSTITUTE OF TECHNOLOGY, TECHNION CITY, HAIFA, ISRAEL

DEPARTMENT OF MATHEMATICS, UNIVERSITY OF HAWAII, HONOLULU, HAWAII 96822

Current address: Department of Mathematics, University of Michigan, Ann Arbor, Michigan 48109 